\title{
Chemical enhancement materials for grounding purposes
}

\begin{abstract}
It is an aim of this work in finding the best performed chemical enhancement material in order to provide the lowest impede path for fault currents to be dispersed into the earth in the shortest time possible. These fault currents are typically due to transients and they could be dangerous to the power system, equipment in it and the personnel involved with the system. Hence, it is desired that a grounding system is installed to provide such path to clear these fault currents from the power system in the manner that danger could be reduced. Such transients are generated by lightning, or switching action. Note that in this study, only lightning strike is considered. Lightning could create impulsive transient during its direct or indirect strikes to power system. This problem is normally prevented by installing a suitable grounding system to the power system. A grounding system will create a path for transient to be safely dispersed into the earth by providing lowest resistance path. This can be easily done using either natural or chemical enhancement materials. However, this work only reported the findings of having chemical enhancement materials in the vicinity of grounding system for protection against lightning. It is found that sodium chloride demonstrated the best performance in being enhancement material for earthing system on top of sodium thiosulphate, magnesium chloride, cuprum sulphate, and ammonium chloride.
\end{abstract}

